\title{
A sensitive, semi-quantitative mammalian two-hybrid assay
}

\author{
Elisabeth Riegel, Thomas Heimbucher ${ }^{\dagger}$, Thomas Höfer ${ }^{\dagger \dagger}$, and Thomas Czerny \\ Department of Applied Life Sciences, University of Applied Sciences, FH Campus Wien, Vienna, Austria
}

†Present address: Lewis-Sigler Institute for Integrative Genomics, Princeton University, Princeton, NJ
†'Present address: Baker IDI Heart and Diabetes Institute, Melbourne, Victoria, Australia

BioTechniques 62:206-214 (May 2017) doi 10.2144/000114544

Keywords: protein-protein interactions; mammalian two-hybrid

Supplementary material for this article is available at www.BioTechniques.com/article/114544.

Protein-protein interactions critically determine the function of a protein within the cell. Several methods have been developed for the analysis of protein interactions, including two-hybrid assays in yeast and mammals. Mammalian two-hybrid systems provide the ideal physiological environment to study the interactions of mammalian proteins; however, these approaches are limited in sensitivity and their ability to quantify interaction strength. Here, we present an inducible mammalian two-hybrid (iM2H) system using the small-molecule dimerizer rapalog for recruitment of multiple transactivation domains into the $\mathrm{M} 2 \mathrm{H}$ system. This inducibility, combined with additional improvements of the $\mathrm{iM} 2 \mathrm{H}$ components, results in an up to 100-fold increase in sensitivity compared with conventional $\mathrm{M} 2 \mathrm{H}$ approaches. In addition, we include a number of reference interactions in our $\mathrm{M} 2 \mathrm{H}$ approach, which enable semiquantitative assessment of protein interactions. Using Groucho/Tle proteins and their binding partners, we demonstrate the applicability of our $\mathrm{iM} 2 \mathrm{H}$ to established protein networks. Finally, to test the applicability of our system for drug screening, the interference of a small-molecule inhibitor on a known proteinprotein interaction was tested, and the particular advantages of the internal reference interactions were shown.

Protein-protein interactions critically influence protein function by defining interaction networks within the cell. A number of methods exist for analysis of protein interactions in mammalian cells (1). Among the cell-based systems, a genetic approach using reporters was accomplished by the invention of the two-hybrid technique (2). This method takes advantage of separate DNA-binding domains (DBDs) and activation domains (ADs), which can be recombined into an active complex by proteinprotein interactions. To analyze two proteins of interest, one is fused to the DBD (termed "bait") and the other to the AD (termed "prey"). A reporter construct containing specific binding sites for the DBD is consequently activated by a functional bait-prey complex and drives expression of a reporter gene.
Originally, this system was developed for application in yeast (yeast two-hybrid) and later adapted for use in mammalian cell culture systems [mammalian two-hybrid $(\mathrm{M} 2 \mathrm{H})](3,4)$. The use of mammalian cells is intended to overcome one of the major limitations of the yeast two-hybrid system by providing mammalian-specific post-translational modifications. The particular strength of the method is therefore the extended analysis of individual protein-protein interactions, including the effects of post-translational modifications and signaling processes, thus providing a fast route to interaction site mapping (1). In recent years, a variety of applications in drug discovery were also developed (5).

Dimerizer-controlled interaction technology is based on the macrolide antibiotic rapamycin from Streptomyces hygroscopius, which bivalently binds to both the intracellular receptor FKBP12 (via the FK domain) and a domain of the FKBPrapamycin associated protein (FRAP). Subsequently, non-toxic derivatives of rapamycin (termed rapalogs) were synthesized in combination with mutated forms of the FRAP domain (termed FR) to suppress the mTor inhibitory function of rapamycin in cells and organisms, and they are used as a tool to heterodimerize chimeric proteins fused to FK and FR (6). One particular application of this system is dimerizer-induced enhancement of transcriptional activators (7).

$\mathrm{M} 2 \mathrm{H}$ provides a highly versatile approach for protein-protein interaction analysis; however, when we tested known interactions, we repeatedly observed a lack of the sensitivity necessary to detect weak interactions. Therefore, we systematically optimized individual components of the $\mathrm{M} 2 \mathrm{H}$ system in

\section{METHOD SUMMARY}

An inducible version of a highly sensitive mammalian two-hybrid system is presented along with extensive control and reference interactions, allowing semiquantitative evaluation of protein-protein interactions. 
an effort to substantially improve sensitivity. The introduction of our inducible transactivation ( $\mathrm{iM} 2 \mathrm{H})$ method, together with a set of reference and control reactions, allows a thorough analysis of protein-protein interactions in a semiquantitative manner.

\section{Materials and methods}

\section{Plasmids}

The reporter plasmid backbone pMlucF for the Gal4 and zinc-finger homeodomain (ZFHD) reporters contains a multiple cloning site upstream of a minimal Fos promoter (8) and the firefly luciferase gene (Fluc). The Renilla luciferase (Rluc) reporters (prefix pMRlucF) were constructed by exchanging Fluc with Rluc from phRL-SV40 (Promega, Madison, WI). For Gal4 binding sites, the consensus sequence CGGAACACTGAAGTCCG was used (bound by two Gal4 proteins) (9) and for ZFHD, the sequence TAATGATGGGCG was used (10) and multimerized up to 48 times. All expression constructs were cloned into the pMC backbone (11). For constitutive expression, the Gaussia luciferase (Gluc) was cloned from pGluc Basic (New England Biolabs, Ipswich, MA) into the pMC expression vector. The Gal4 DBD contains amino acids 1-147 (12). The ZFHD DBD and NFk-B p65 AD sequences were isolated by PCR from pHet-Act1-1 (Takara Bio USA, Mountain View, CA) and cloned into pMC expression vectors. 3xFK (FKBP12) and FR (FRAP, containing the mutation T2098L) were also cloned from pHet-Act1-1. The following bait and prey components were cloned by PCR: Lef1, Blimp1, Pax5, Gbx2, Otx2, En2, Hes1, b-catenin, Rb, Tle4, p53, and MDM2. Sequences for the acid and base peptides (13) and base peptide mutants as well as P1 (14) were generated by PCR. Sequences for the internal reference interaction proteins MinD and MinE were isolated from E. coli genomic DNA with PCR. For details see Supplementary Table S1.

\section{Rapalog system}

For rapalog induction, cells were grown for $24 \mathrm{~h}$ after transfection before medium was removed and replaced with $100 \mu$ freshDMEM supplemented with 10\% FCS, penicillin/ streptomycin (pen/strep), and 33 nM rapalog (A/C Heterodimerizer \#635057; Takara Bio USA). Cells were incubated for an additional $24 \mathrm{~h}$ before luciferase measurement.

Cell culture and transfection

HeLa cells were grown in DMEM with 4.5 $\mathrm{g} / \mathrm{L}$ glucose, $10 \%$ FCS, and $1 \times$ pen/strep.
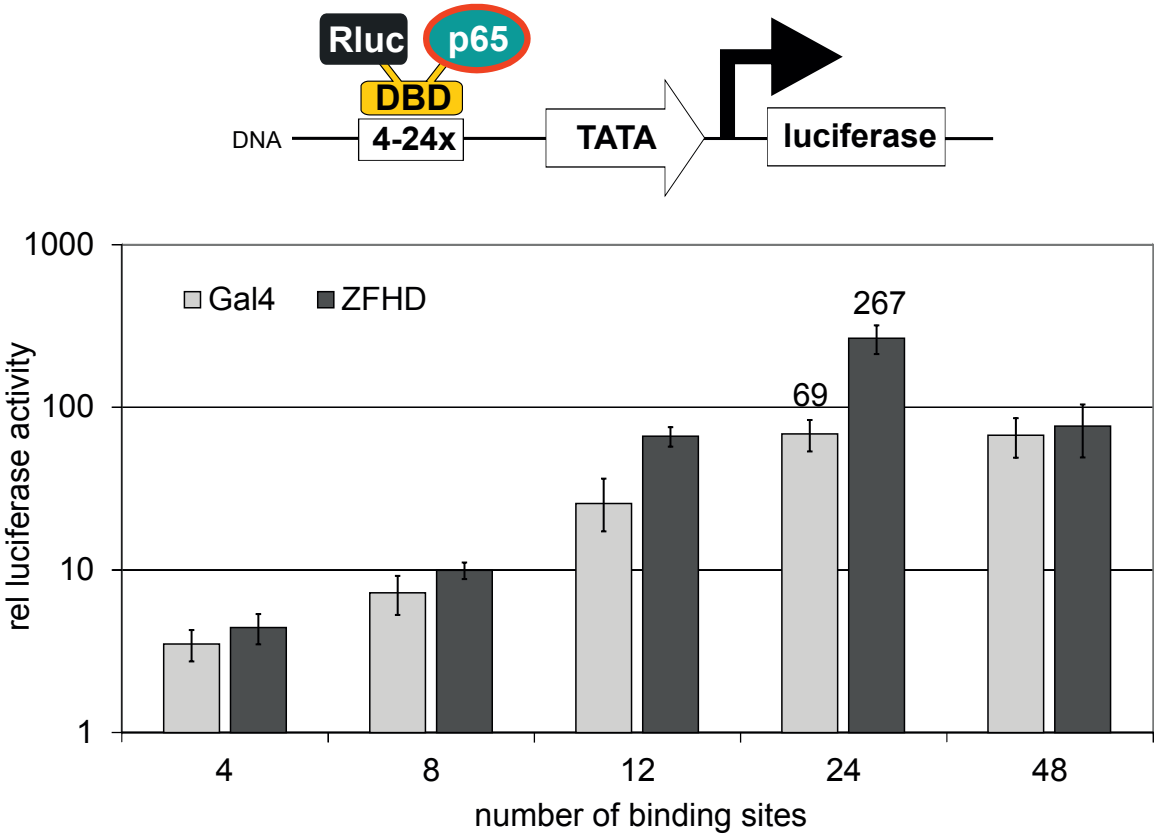

Figure 1. DNA-binding domain (DBD) and reporter construct optimization. Reporter plasmids contain 4-48 individual binding sites for either ZFHD or Gal4 (corresponding to 2-24 dimeric Gal4 binding sites) upstream of a minimal promoter and Fluc. Cells were transfected with reporter plasmid and $2 \mathrm{ng}$ of an activator plasmid containing either the DBD of ZFHD (pMCRluc-ZF-65) or that of Gal4 (pMCRlucGal4-65) fused to the p65 activation domain (AD) and Rluc. The $y$-axis shows relative luciferase activity as Fluc/Rluc relative to an empty reporter control without any binding site (pMlucF). All data are the mean of at least three independent experiments containing six replicates each. Error bars indicate SE.

For transient transfection experiments, $3 \times$ $10^{3}$ cells were seeded in a polyethyleneimine (PEI)-coated (15) transparent 96-well plate, incubated for $24 \mathrm{~h}$ at $37^{\circ} \mathrm{C}$, and transfected with TurboFect (Thermo Fisher Scientific, Waltham, MA) according to the manufacturer's instructions. If not stated otherwise, 90 ng of reporter plasmid was used for transfection in single-reporter assays, and $40 \mathrm{ng}$ was used for two-reporter assays. For $\mathrm{iM} 2 \mathrm{H}$ experiments, 2 ng of bait constructs, 20 ng of prey constructs, and $20 \mathrm{ng}$ of pMCFR-65 were transfected. If a constitutive internal reference was used, 2 ng of pMCGlucS was transfected. All constructs were transfected as six replicates. For experiments without rapalog induction, a luciferase assay was performed $24 \mathrm{~h}$ after transfection; for those with rapalog induction the assay was done $48 \mathrm{~h}$ after transfection.

\section{Dual luciferase assay and} statistical analysis

For dual luciferase activity determination, cells were washed once with $1 \times$ PBS and lysed in $25 \mu$ lysis buffer $(25 \mathrm{mM}$ Tris $\mathrm{pH}$ 7.5, 0.03\% Triton X-100), and luciferase activity measurement was performed with a Thermo Scientific Luminoskan Ascent Microplate Luminometer (Thermo Fisher Scientific) with 3 injectors. The dual luciferase program consisted of a $40-\mu$ injection of firefly substrate [6.25 mM Tris pH 7.5, 10 mM MgCl, $100 \mu \mathrm{M}$ D-luciferin (Synchem, Felsberg/Altenberg, Germany), 2.5 mM ATP] and a measurement step, followed by injection of $40 \mu \mathrm{l}$ Rluc or Gluc substrate [6.25 mM Tris pH 7.5, $210 \mathrm{mM}$ Titriplex IV, $3 \mu \mathrm{M}$ coelenterazine (Synchem)] and measurement after a $16 \mathrm{~s} \mathrm{lag}$ time. Finally, $100 \mu \mathrm{l} \mathrm{0.3} \mathrm{M} \mathrm{H}_{2} \mathrm{SO}_{4}$ was injected to stop luciferase reactions before proceeding to the next well. For normalization, the Fluc relative light unit $(R L U)$ values were divided by those for Rluc or Gluc. P-values were calculated by a two-tailed Student's t-test in Microsoft Excel.

\section{p53-Mdm2 inhibition assay}

Nutlin-3 (\#sc-45061; Santa Cruz Biotechnology, Dallas, TX) was diluted in DMEM supplemented with 10\% FCS, pen/strep and $33 \mathrm{nM}$ rapalog from a $10 \mathrm{mM}$ stock in DMSO. Twenty-four hours after transfection, medium was removed, and $100 \mu \mathrm{l}$ DMEM/rapalog/ Nutlin-3 was added to the cells. Viability and dual luciferase assays were performed $24 \mathrm{~h}$ after rapalog and Nutlin-3 addition. For determination of the $\mathrm{IC}_{50}$ the four-parameter logistic function was used:

$y=\min +(\max -\min ) /\left(1+10^{((x-\log / C 50) * \text { slope })}\right)$

with $\mathrm{x}$ in logarithmic form, and curve fitting was done using the least-squares method. 
A „conventional“ M2H

\section{no interaction}
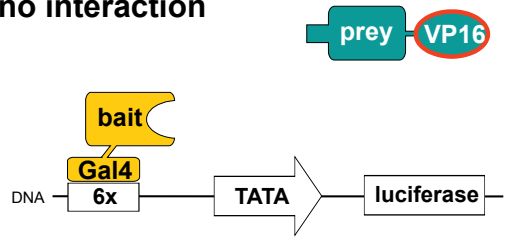

interaction

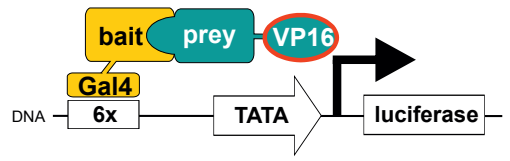

B

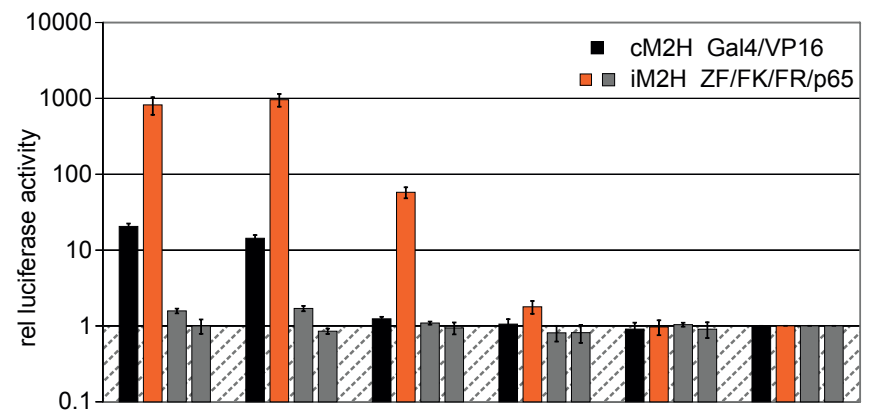

bait base base[-6] base[-7] base[-9] base[-10] lamin

rapalog + - + - + - + + + + + +

prey (acid) $+++-+++-+++-+++-\quad+++-+++-$

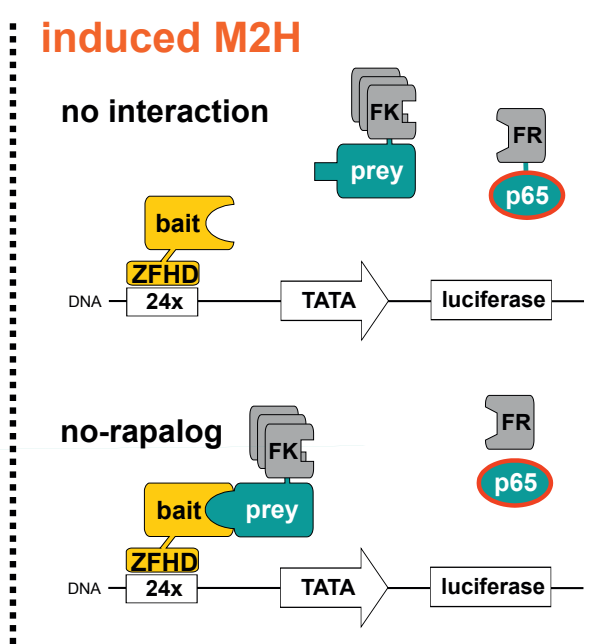

Figure 2. Inducible mammalian two-hybrid (iM2H) system. (A) For comparison of the conventional $\mathrm{M} 2 \mathrm{H}$ (cM2H) (Gal4/VP16; left side) to the iM2H (ZF/FK/ FR/p65; right side), the artificial leucine zipper interactions of the acid and base peptides and truncations of the base peptide (left panel) and the interactions of Lef1 with $\beta$-catenin and Rb with the peptide P1 (right panel) were analyzed. For cM2H (black bars), $70 \mathrm{ng}$ of Gal4 reporter plasmid (pMlucF 6Gal) was co-transfected with $20 \mathrm{ng}$ of the indicated bait protein constructs and $20 \mathrm{ng}$ of the prey protein construct (pMC-acid-VP16, pMC- $\beta$ cat $\Delta-V P 16$, pMC$\mathrm{P} 1-\mathrm{VP16}$ ). For iM2H (orange bars), a zinc-finger homeodomain (ZFHD) reporter plasmid (pMlucF 24ZF) was co-transfected with $2 \mathrm{ng}$ of the indicated bait protein constructs, 20 ng prey protein constructs (pMCaFK, pMC- $\beta$-cat $\Delta-F K, p M C-P 1-F K$ ), and 20 ng activator construct (pMCFR-65). For iM2H, two control reactions were performed (gray bars): one without the addition of rapalog and one without the addition of prey protein. pMC GlucS was co-transfected for internal normalization. The $y$-axis in (B) shows relative luciferase activity as Fluc/Gluc normalized to the negative bait control (lamin). In (C), cM2H is normalized to the negative bait control (lamin) and iM2 $\mathrm{H}$ to the reporter without addition of bait/prey constructs. All values are the mean of at least three independent experiments with six replicates each. Error bars indicate SE.

\section{Viability measurements}

For viability measurement, resazurin (Santa Cruz Biotechnology) (16) was added to the medium to a final concentration of $50 \mu \mathrm{M}$. Relative fluorescence (excitation at $544 \mathrm{~nm}$, emission at $590 \mathrm{~nm}$ ) was measured after 2 $\mathrm{h}$ incubation at $37^{\circ} \mathrm{C}$.

\section{Results and discussion}

\section{Basic setup of the $\mathrm{M} 2 \mathrm{H}$ assay}

The yeast Gal4 transcription factor is most widely used for the DBD of the bait in the $\mathrm{M} 2 \mathrm{H}$ assay. It binds as a dimer to 17-bp palindromic binding sites (17), and typical reporter constructs contain 5 or 6 dimeric binding sites (18). To test whether this represents an optimized number for reporter sensitivity, we introduced up to $48 \mathrm{Gal} 4$ binding sites into the firefly luciferase (Fluc) reporter construct (corresponding to 24 dimeric consensus binding sites). The reporter was activated by co-transfection of an artificial Gal4 transcription factor construct (pMCRlucGal4-65) in which the DBD of Gal4 is fused both to the AD of VP16 and to Renilla luciferase (Rluc). This allows normalization of the reporter activity (Fluc signal) to the protein level of the activator in the cells (Rluc signal). We observed increasing reporter activity (Fluc/Rluc) for constructs with higher numbers of binding sites (Figure 1). Eight and 12 binding sites (corresponding to 4 and 6 dimeric sites, respectively) resulted in 4- to 22-fold activation compared with a reporter control lacking Gal4 binding sites. However, the strongest activity was observed with 24 consensus sequences (69-fold), while 48
Gal4 binding sites did not further increase promoter activity.

We next tested the ZFHD fusion protein, which has a DBD that strongly and selectively binds to a ZFHD-composite DNA element (10). For direct comparison with the Gal4 system, we fused the ZFHD to both the AD of p65 and Rluc (pMCRluc-ZF-65) and used comparable reporters containing 2-48 ZFHD binding sites. Again, maximum activity was obtained for 24 binding site repeats (Figure 1); however, the ZFHD system was clearly more active ( 270 fold) compared with induction levels observed for Gal4 ( 70 fold; $P=0.034)$. Due to the normalization to Rluc values, differences in the expression levels or protein stability of the different activator constructs can be excluded. In order to extend the comparison, titration of different 
amounts of each of the 2 DBD/AD constructs relative to a constant amount of the corresponding reporter plasmid containing 24 binding sites was carried out. This demonstrated higher activity of the ZFHD system over a broad range of concentrations (Supplementary Figure S1), thus indicating differences in the affinity of the DBDs for their respective binding sites.

Since both the Gal4 as well as the ZFHD system showed reduced activity with 48 binding sites, we tested whether promoter length is the limiting factor. Different promoter lengths were compared by positioning identical binding sites at variable distances upstream of the transcription initiation site (Supplementary Figure S2). Indeed, we found an almost linear decrease in the contribution of binding sites depending on their distance, reaching zero activation of the promoter in the range of $800 \mathrm{bp}$. Twenty-four ZFHD binding sites cover 432 bp upstream of the transcription start. Consequently, the decrease in activity for 48 binding sites (864 bp) can be explained by their greater distance from the start site. In this case, the contribution of the more distant sites is negligible, but they nevertheless compete with more proximal sites for ZFHD binding, resulting in reduced activity. Taken together, these results reveal that the newly designed ZFHD reporter incorporating 24 binding sites has $>10$-fold higher activity compared with a conventional Gal4 reporter containing 6 dimeric binding sites.

\section{Establishment of an inducible} mammalian two-hybrid (iM2H) system The conventional $\mathrm{M} 2 \mathrm{H}(\mathrm{cM} 2 \mathrm{H})$ system (Figure 2A, left panel) usually features reporter plasmids with five or six dimeric Gal4 consensus binding sites upstream of a minimal promoter (TATA). The AD fused to the prey protein is typically the viral protein VP16, the prototype of a strong activator. Attempts to further potentiate the activity of the AD by multimerization were only partially successful, as strong activators are rapidly degraded in the cell, especially when actively engaged in transcriptional activation (19). However, this limitation can be circumvented by using separated domains that are expressed independently and recombine only upon addition of a small-molecule dimerizer. This results in hyper-transactivation (7). The inducible system is based on the dimerizer function of the rapamycin derivative rapalog (A/C Heterodimerizer), which links the FK and FR domains. We applied this method to the $\mathrm{M} 2 \mathrm{H}$ system and established an inducible $\mathrm{M} 2 \mathrm{H}$ (iM2H) approach based on the ZFHD system (Figure 2A, right panel). We separated the prey into a prey-FK fusion and an FR-AD fusion. Upon addition of rapalog, the FK and FR domains dimerize and thus combine the AD with the prey. A specific advantage of our system is that several FK domains can be added to the prey, and subsequently several ADs can be attached to one prey molecule (Supplementary Figure S3A). Here, we found an optimal activation of iM2H with three FK domains (Supplementary Figure S3B). In addition, we tested different ADs and found the p65 domain to be more effective than VP16 (Supplementary Figure $\mathrm{S3C)}$.

For accurate assessment of the sensitivity of the two-hybrid assays, a reliable set of reference interactions covering a wide range of affinities is required. For this purpose the Velcro peptides consisting of two artificial leucine zipper domains (13) were adopted. They include glutamic acid in one peptide termed "acid" and lysine in the other peptide termed "base" (Supplementary Figure S4). The resulting electrostatic interactions stabilize heterodimers but prevent homodimer formation. The peptides are small (30 amino acids) but mediate a robust and highly specific interaction with $a K_{d}$ of $30 \mathrm{nM}$ (13). By stepwise deletion of amino acids from the $\mathrm{C}$ terminus of the base peptide (base[-6] to base[-10]), we could generate a versatile set of reference interactions with decreasing affinity that could be used for M2H (Supplementary Figure S4).

Using this acid-base peptide reference interaction system, we directly compared the sensitivities of $\mathrm{iM} 2 \mathrm{H}$ and $\mathrm{CM} 2 \mathrm{H}$ (Figure 2B). iM2H showed almost 100-fold higher reporter induction (orange bars) compared with the Gal4 system (black bars). The largest differences were seen for the strong interaction of base[-6] (14-fold and 940-fold activation for $\mathrm{cM} 2 \mathrm{H}$ and $\mathrm{iM} 2 \mathrm{H}$, respectively; $P=0.0008$ ). Furthermore, the base[-7] interaction was hardly detectable by $\mathrm{cM} 2 \mathrm{H}$ (1.2-fold induction) but resulted in a robust interaction signal in $\mathrm{iM} 2 \mathrm{H}$ (57-fold induction; $P$ $=0.0043)$. The base[-9] interaction revealed a weak activation with iM2H (1.8-fold induction), whereas base[-10] did not differ from the lamin controls, which serve as a control to detect sticky proteins (20).

Increasing the sensitivity is one major way to prevent false-negative results; however, false-positive reactions are also a major problem with $\mathrm{M} 2 \mathrm{H}$. But the introduction of dimerizer-induced transactivation provides ideal negative control conditions by omitting the dimerizer (no-rapalog control). In this case, the bait-prey complex is fully formed on the DNA, but still lacks the AD; thus, any potential activating or repressing functions of the interaction partners can be detected. The no-rapalog controls for the acid-base interaction were all close to background induction levels (gray bars in Figure 2B).

We extended the experiments to other well-documented protein-protein interactions. The interaction of $\beta$-catenin with Tcf/ Lef transcription factors is essential for Wnt signaling (21), and an interaction of the tumor suppressor retinoblastoma protein $(\mathrm{Rb})$ with the artificial peptide P1 was identified in a yeast two-hybrid screen (14). Both interactions were efficiently detected by $\mathrm{iM} 2 \mathrm{H}$ (250-fold and 36-fold, respectively), whereas the reporter signals in $\mathrm{CM} 2 \mathrm{H}$ were low, particularly for the Rb-P1 interaction, with values close to the basal level (1.8-fold) (Figure 2C). The no-rapalog control of $\beta$-catenin revealed detectable activation (4.9-fold), which can be explained by the transactivating properties of $\beta$-catenin. Furthermore, the controls with lamin displayed weak background activation, indicating the slightly sticky properties of $\beta$-catenin. In order to evaluate the potential of $\mathrm{iM} 2 \mathrm{H}$ to detect interactions for non-nuclear proteins, we tested the membraneassociated protein E-cadherin and found a robust interaction with $\beta$-catenin (26-fold activation; experimental design equivalent to that depicted in Figure 2C). We found that the induction values of $\mathrm{cM} 2 \mathrm{H}$ are low for the biologically relevant $\beta$-catenin-Lef1 interaction, whereas our iM2H displayed strong activation and very sensitive detection for this interaction; however, the controls indicate an overestimation of the interaction strength when directly compared with reference interactions.

\section{Internal reference interaction}

As the example of $\beta$-catenin demonstrates, the no-rapalog controls of $\mathrm{iM} 2 \mathrm{H}$ represent a considerable improvement for evaluation of the results; however, an ideal control for a two-hybrid assay would be a reference protein-protein interaction performed within the same cells. In order to establish such a control, we selected the MinD and MinE proteins of E. coli, which represent an established protein-protein interaction (22). A small peptide of MinE (35 amino acids) is sufficient for this interaction and can be fused to the prey of the test interaction. The 
A

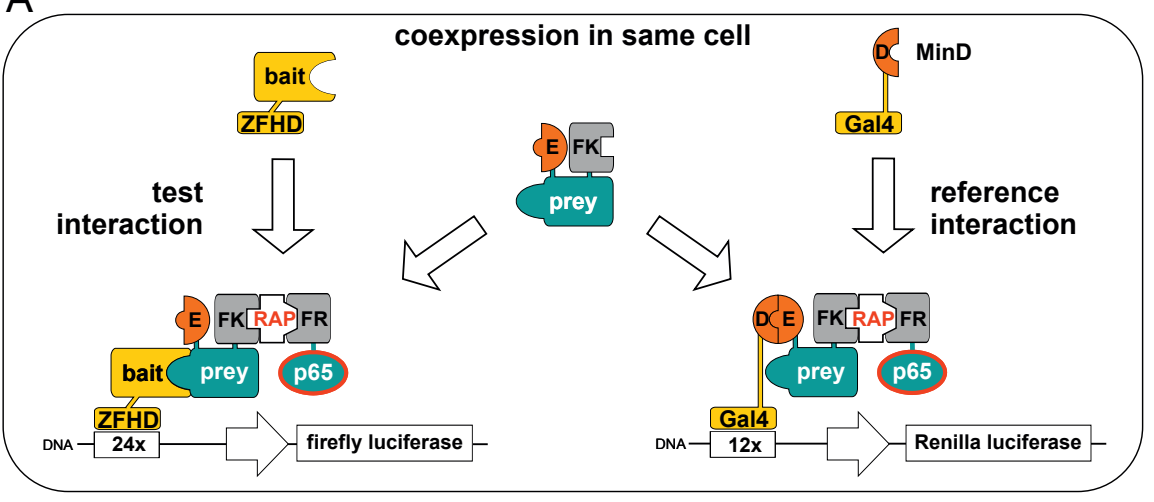

B

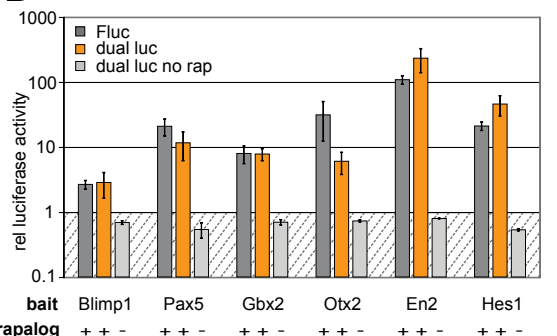

C

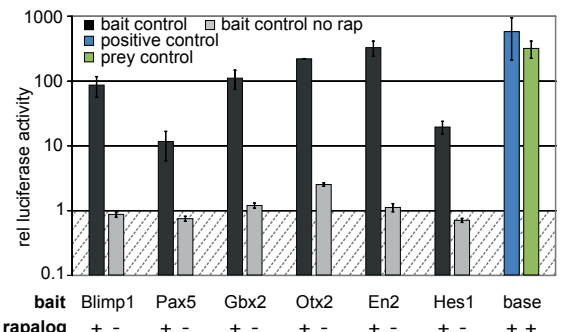

Figure 3. Reference and control reactions for the quantification of protein-protein interactions in the inducible mammalian two-hybrid (iM2H) system. For internal normalization, a dual reporter system (A) is co-expressed within the same cell. A Fluc reporter and the zinc-finger homeodomain (ZFHD) system were used to detect the test interaction, whereas a Rluc reporter and the Gal4 system were used to detect the internal reference interaction of MinD with MinE ( $D$ and $E$ ). The prey construct functions in both reporter systems since it is a fusion of the prey protein-of-interest, the internal reference prey MinE, and three FK domains (of which only one is shown). (B) Cells were transfected with the test reporter ( $\mathrm{MMlucF}$ 24ZF) and reference reporter (pMRlucFpA 12Galn), bait constructs containing Blimp1, Pax5, Gbx2, Otx2, En2, or Hes1, and prey constructs expressing Tle4 and MinE (for the internal reference interaction) together with reference bait construct (pMCGal4-MinD) and 20 ng pMCFR-65. (C) Same as in (B) except that the bait constructs contained fusions with the base[-6] peptide (bait control), and prey constructs contained acid peptide but no Tle4 (pMCeaFK; except for the prey control, which includes Tle4). As a positive control and prey control, base[-6] peptide constructs were transfected. The $y$-axis shows relative luciferase activity as Fluc (dark gray bars in Panel B) or Fluc/Rluc (dual luc; all except dark gray bars in Panel B) relative to the lamin control. All values are the mean of at least three independent experiments with six replicates each. Error bars indicate SE.

resulting protein therefore has a dual role in serving as a prey for both the reference and the test interaction (Figure 3A). To detect the two interactions independently, the reference interaction uses a separate reporter. For this purpose, we used the Gal4 system and Rluc for detection. During the experiments, both the reference and the test interaction are induced by rapalog addition, which simultaneously activates the prey components, generating highly similar conditions for both reactions. Consequently, Fluc activity (test interaction) and Rluc activity (internal reference interaction) can be detected simultaneously in a dual luciferase assay.

We first tested whether the two interactions could function independently from one another. In particular, the dual role of the prey component could affect the efficiency of binding to the two different baits; however, we could not detect any interference between the two reactions, indicating that the prey protein is not limiting in the cells under the assay conditions. In order to evaluate the system, we performed a number of $\mathrm{M} 2 \mathrm{H}$ binding tests using known Gro/Tle protein interactions. The family member Tle4 was used as prey, and the interaction partners Blimp1, Pax5, Gbx2, Otx2 En2, and Hes1 were used as baits. In good agreement with the literature (23-27), the Gro/Tle interactions could be detected, and the no-rapalog controls support the specificity of the signals (Figure 3B). The presence of MinE in the prey protein did not affect these interactions (Supplementary Figure S5). We next compared the results with and without calculation of the internal reference (Figure 3B; Fluc versus dual luc). The signals for the 2 conditions only slightly differed, except for the Otx2-Tle4 interaction, which appeared markedly reduced (from 31-fold to 6-fold) after correction with the internal reference interaction. Therefore, in the case of Otx2, the internal reference prevents an overestimation of the interaction strength.

As shown for $\beta$-catenin, bait or prey proteins can substantially influence the transcriptional activity of the complexes and lead to quantitative misinterpretation of the $\mathrm{M} 2 \mathrm{H}$ assay. We therefore extended the controls by applying the acid-base peptide system. The interaction of the base[-6] and acid peptides served as a positive control (ZF-base[-6] + acid-FK; blue bar in Figure 3C). Although Tle4 is an established co-repressor, its presence in the prey control only slightly affected the read out (ZF-base[-6] + acidTle4-FK; prey control; green bar in Figure $3 \mathrm{C})$, indicating that the repressing activity of Tle4 is strongly overruled by the AD of multimerized p65 and therefore does not dramatically affect the evaluation of the interaction strength. In another experiment, the bait constructs were fused with the base[-6] peptides. The presence of the baits resulted in reporter activities in the same range as the positive control, except for Pax5 and Hes1, which were markedly reduced in this assay (ZF-bait-base[-6] + acid-FK; bait control; Figure 3C, black bars). These two proteins are known repressors $(28,29)$, and the controls indicate that part of this repression activity is Gro/Tle-independent and affects the $\mathrm{iM} 2 \mathrm{H}$ readout. Taken together, the controls suggest an underestimation of the Pax5 and Hes1 interactions and an overestimation of those involving Otx2. Applying the internal reference interaction and the no-rapalog, bait, and prey controls allow a thorough crosscheck of the two-hybrid results in a single experiment. In summary, the semiquantitative results of the $\mathrm{iM} 2 \mathrm{H}$ analysis of the Gro/Tle protein interactions are: strong interactions of Tle4 with En2, Hes1, and Pax5, intermediate interactions with Otx2 and Gbx2, and weak interactions with Blimp1.

The $\mathrm{iM} 2 \mathrm{H}$ assay in drug discovery The biological importance of protein-protein interactions in cellular signaling makes them an attractive target for therapeutic intervention. However, the development of drugs interfering with protein-protein interactions presents a major challenge. $\mathrm{M} 2 \mathrm{H}$-based bioassays are an attractive screening strategy, in particular when posttranslational modifications of the proteins are critical. However, these screenings are hampered by a large number of falsepositive hits due to the high concentrations of low-potency compounds during the first 

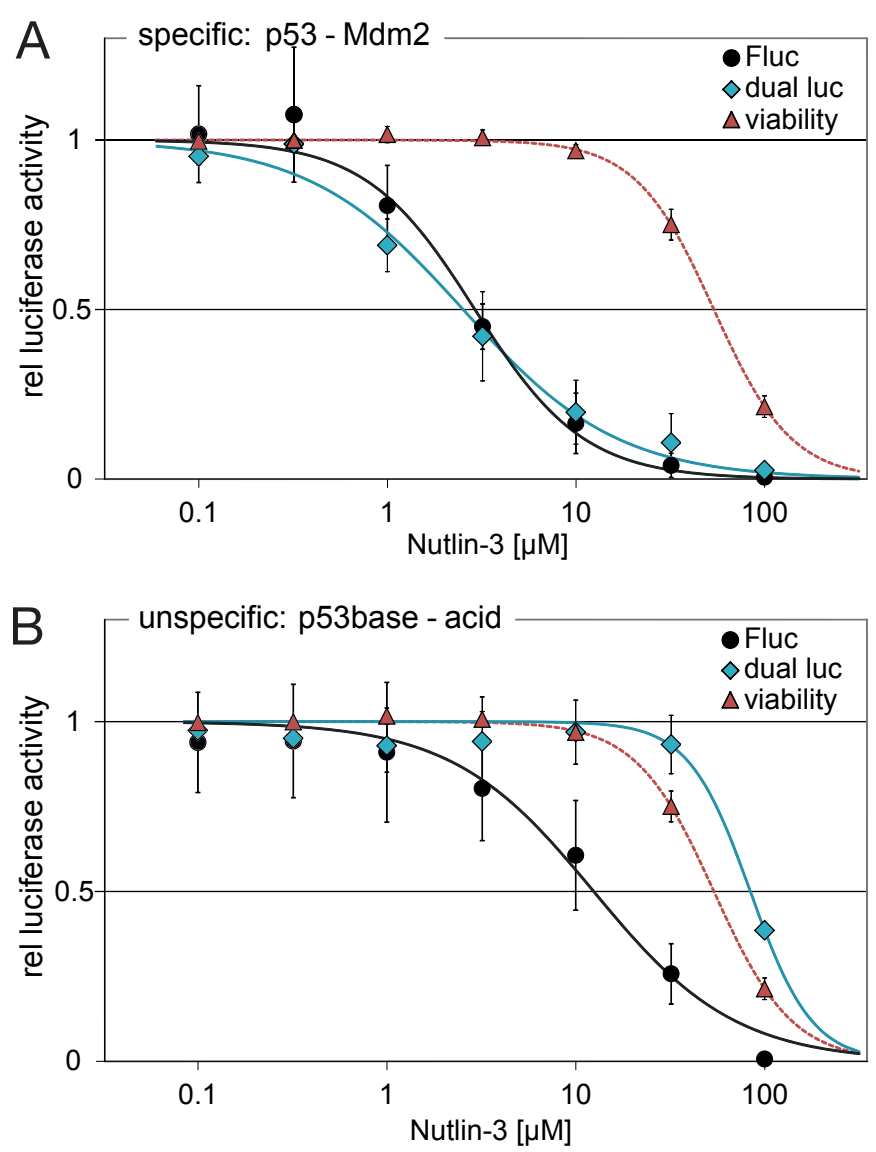

Figure 4. Analysis of Nutlin-3 inhibition of the p53-Mdm2 interaction using the inducible mammalian two-hybrid (iM2H) system. For the application of $\mathrm{iM} 2 \mathrm{H}$ to drug screening, cells were transfected with test reporter (pMlucF 24ZF), internal reference reporter (pMRlucFpA 12Galn), bait construct coding for p53 (A) or p53 (amino acids 13-28) fused to base[-6] peptide (pMCZF-base[-6]-p53(13-28)) (B), and a prey construct coding for Mdm2 (amino acids 25-113) (A) or acid peptide (pMCeaFK) (B), together with internal reference bait (pMCGal4-MinD) and pMCFR-65. Twenty-four hours after transfection, cells were treated with rapalog and Nutlin-3 and analyzed $24 \mathrm{~h}$ later. The $y$-axis shows relative luciferase activity as Fluc (black dots), Fluc/ Rluc (blue squares), or relative viability (red triangles), always compared with cells without Nutlin-3 addition. All values are the mean of at least three independent experiments with six replicates each. Error bars indicate SE.

screening rounds (30). To test the application of the $\mathrm{iM} 2 \mathrm{H}$ system and the internal reference interaction for drug screening, we used the p53-Mdm2 interaction and the small molecule Nutlin-3 as a specific inhibitor of this interaction (31). Using p53 as bait and Mdm2 as prey, we obtained a robust interaction in the $\mathrm{M} 2 \mathrm{H}$ ( 1200 -fold activation). Application of increasing amounts of Nutlin-3 reduced the two-hybrid activity starting at $1 \mu \mathrm{M}$ (Figure 4A). The toxicity of the drug was monitored by measuring the viability of the cells with resazurin. Using the dual luciferase measurements based on the internal reference interaction, we calculated an $\mathrm{IC}_{50}$ of $2.5 \mu \mathrm{M}$ for Nutlin-3, in good agreement with the literature (31). Calculating the $\mathrm{IC}_{50}$ concentration using only the Fluc values (without internal reference) resulted in 2.9 $\mu \mathrm{M}$, which is almost identical to that for the dual luciferase variant (compare Fluc and dual luc in Figure 4A). In the case of the p53Mdm2 interaction, since the inhibition by Nutlin-3 appears at concentrations not affecting cell viability, the internal reference therefore does not show clear advantages, except for lower variations of the measurements (compare the error bars for the two curves in Figure 4A). However, for an unrelated interaction not affected by

\section{COMING SOON...}
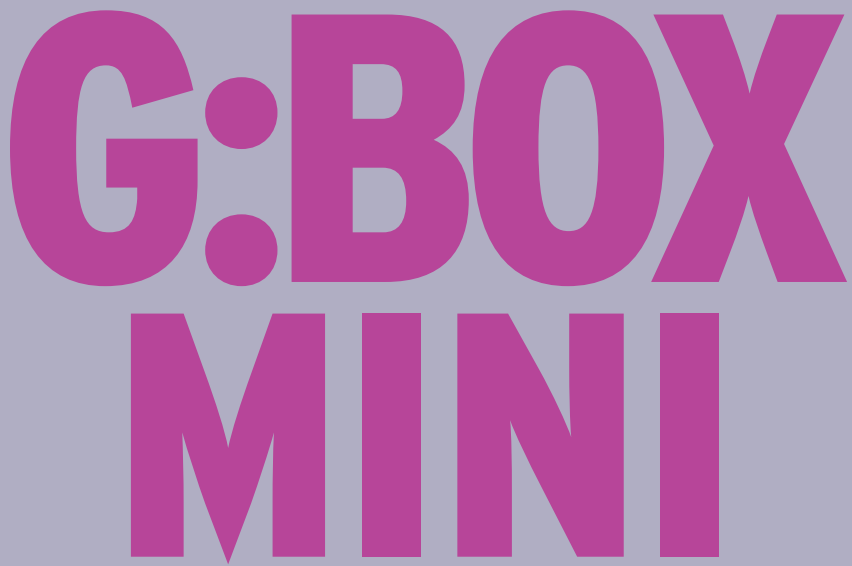

\section{FOR ALL YOUR APPLICATION NEEDS}

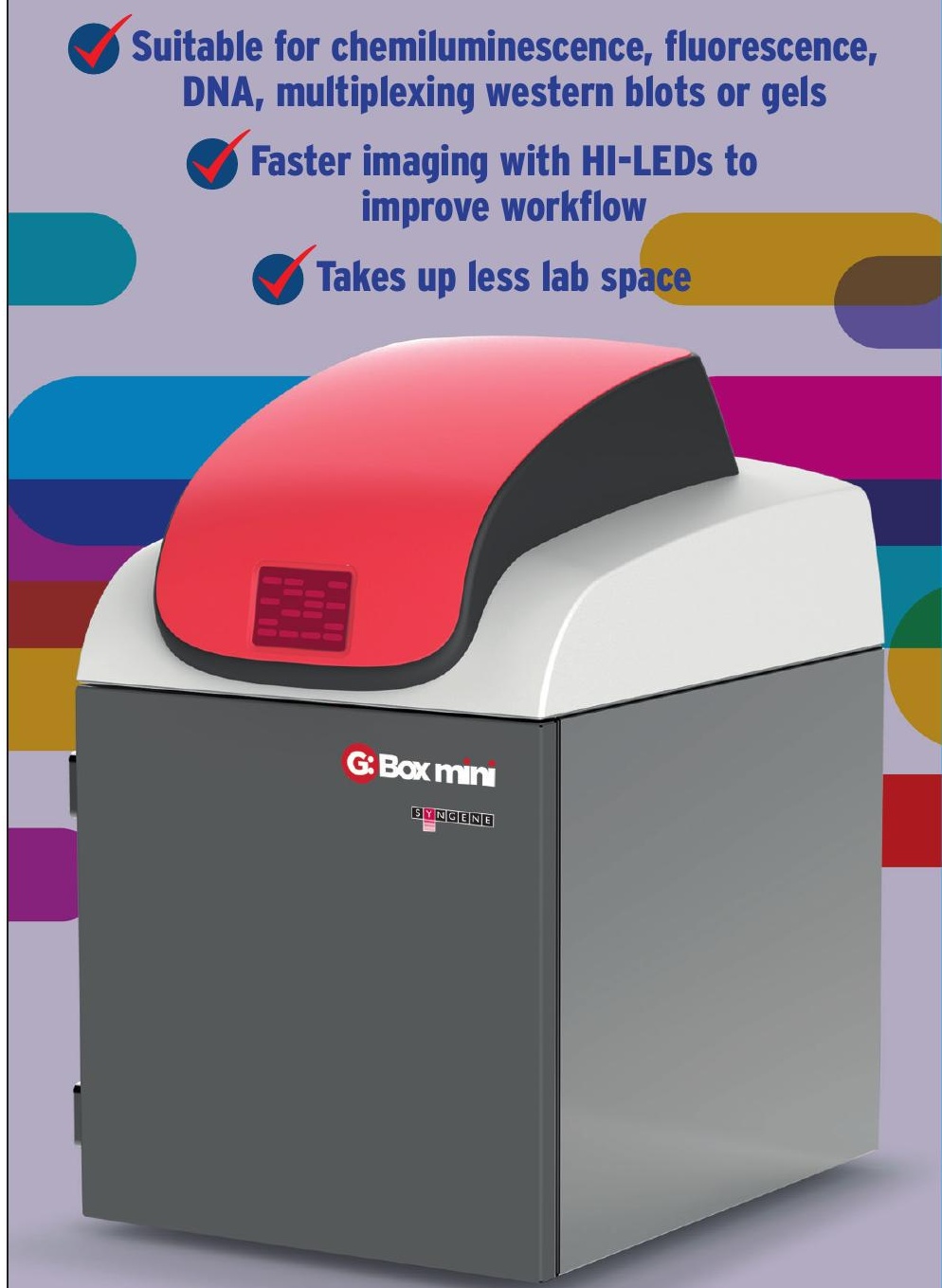

For further information, please contact ussales@syngene.com WWW.SYHGEDG.COM $S|Y| N|G| E|N| E$ 
Nutlin-3, the difference becomes apparent (Figure 4B; acid-base peptide interaction). Using luciferase measurements, a specific inhibitory effect of Nutlin-3 on the acid-base peptide interaction with an $\mathrm{IC}_{50}$ of $12.5 \mu \mathrm{M}$ is suggested (Figure 4B, Fluc). Using the internal reference, the false interpretation of this result becomes obvious (Figure 4B, dual luc; $\left.I_{50}=84 \mu \mathrm{M}\right)$. The $\mathrm{IC}_{50}$ using the internal reference appears in a severely toxic range ( $70 \%$ dead cells), invalidating the significance of this calculation. However, this is not the case for the $\mathrm{IC}_{50}$ of the non-corrected luciferase calculation ( $\sim 5 \%$ dead cells), which therefore might be falsely considered as a significant result. Therefore, this example demonstrates that the internal reference interaction of the $\mathrm{iM} 2 \mathrm{H}$ helps to rule out falsepositive results for small molecules affecting protein-protein interactions.

$\mathrm{iM} 2 \mathrm{H}$ provides a number of advantages over conventional $\mathrm{M} 2 \mathrm{H}$ systems. First, optimization of the reporter, the DBD, and the AD greatly improve the sensitivity of the system (Figure 2 and Supplementary Figure S4). Second, dimerizer induction further enhances the transcriptional activity and provides convenient control reactions (no-rapalog), which define the basal level and detect the effects of bait-prey complexes on transcription. Third, a combination of reference interactions using the acid-base peptides and a number of control reactions allows a semiquantitative evaluation of binding strength. Fourth, an internal reference interaction that also depends on protein-protein interactions allows analyses under increasingly toxic conditions, which is important for overexpression of proteins and for drug screening applications. $\mathrm{iM} 2 \mathrm{H}$ is therefore ideal for highly sensitive detection of proteinprotein interactions and, by using control and reference interactions, the technique provides reliable semiquantitative results.

\section{Author contributions}

E.R. designed and performed all experiments, analyzed data, and wrote the manuscript. T.He. and T.Hö. generated and validated initial constructs and revised the manuscript. T.C. was responsible for experimental design, analysis, writing, communication, and supervision of the project.

\section{Acknowledgments}

We thank Meinrad Busslinger for providing Blimp 1. The work was supported by the City of Vienna (MA23 - project 18-18).

\section{Competing interests}

The authors declare no competing interests.

\section{References}

1. Lievens, S., I. Lemmens, and J. Tavernier. 2009. Mammalian two-hybrids come of age. Trends Biochem. Sci. 34:579-588.

2. Fields, S. and O. Song. 1989. A novel genetic system to detect protein-protein interactions. Nature 340:245-246.

3. Dang, C.V., J. Barrett, M. Villa-Garcia, L.M. Resar, G.J. Kato, and E.R. Fearon. 1991. Intracellular leucine zipper interactions suggest c-Myc hetero-oligomerization. Mol. Cell. Biol. 11:954-962.

4. Vasavada, H.A., S. Ganguly, F.J. Germino, Z.X Wang, and S.M. Weissman. 1991. A contingent replication assay for the detection of proteinprotein interactions in animal cells. Proc. Natl. Acad. Sci. USA 88:10686-10690.

5. Lievens, S., M. Caligiuri, N. Kley, and J. Tavernier. 2012. The use of mammalian two-hybrid technologies for high-throughput drug screening. Methods 58:335-342.

6. Pollock, R. and T. Clackson. 2002. Dimerizerregulated gene expression. Curr. Opin. Biotechnol. 13:459-467.

7. Natesan, S., E. Molinari, V.M. Rivera, R.J. Rickles, and M. Gilman. 1999. A general strategy to enhance the potency of chimeric transcriptional activators. Proc. Natl. Acad. Sci. USA 96:1389813903.

8. Hsu, S.C., J. Galceran, and R. Grosschedl. 1998. Modulation of transcriptional regulation by LEF-1 in response to Wnt-1 signaling and association with beta-catenin. Mol. Cell. Biol. 18:48074818.

9. Liang, S.D., R. Marmorstein, S. Harrison, and M. Ptashne. 1996. DNA sequence preferences of GAL4 and PPR1: how a subset of Zn2 Cys6 binuclear cluster proteins recognizes DNA. Mol. Cell. Biol. 16:3773-3780.

10. Pomerantz, J.L., C.O. Pabo, and P.A. Sharp. 1995. Analysis of homeodomain function by structure-based design of a transcription factor. Proc. Natl. Acad. Sci. USA 92:9752-9756.

11. Fink, M., G. Flekna, A. Ludwig, T. Heimbucher, and T. Czerny. 2006. Improved translation efficiency of injected mRNA during early embryonic development. Dev. Dyn. 235:3370-3378.

12. Keegan, L., G. Gill, and M. Ptashne. 1986. Separation of DNA binding from the transcriptionactivating function of a eukaryotic regulatory protein. Science. 231:699-704.

13. O'Shea, E.K., K.J. Lumb, and P.S. Kim. 1993. Peptide "Velcro": design of a heterodimeric coiled coil. Curr. Biol. 3:658-667.

14. Yang, M., Z. Wu, and S. Fields. 1995. Proteinpeptide interactions analyzed with the yeast two-hybrid system. Nucleic Acids Res. 23:11521156.

15. Vancha, A.R., S. Govindaraju, K.V.L. Parsa, M. Jasti, M. González-García, and R.P. Ballestero. 2004. Use of polyethyleneimine polymer in cell culture as attachment factor and lipofection enhancer. BMC Biotechnol. 4:23.

16. O'Brien, J., I. Wilson, T. Orton, and F. Pognan. 2000. Investigation of the Alamar Blue (resazurin) fluorescent dye for the assessment of mammalian cell cytotoxicity. Eur. J. Biochem. 267:5421-5426.

17. Carey, M., H. Kakidani, J. Leatherwood, F. Mostashari, and M. Ptashne. 1989. An amino- terminal fragment of GAL4 binds DNA as a dimer. J. Mol. Biol. 209:423-432.

18. Lievens, S., I. Lemmens, T. Montoye, S. Eyckerman, and J. Tavernier. 2006. Two-hybrid and its recent adaptations. Drug Discov. Today. Technol. 3:317-324.

19. Molinari, E., M. Gilman, and S. Natesan. 1999. Proteasome-mediated degradation of transcriptional activators correlates with activation domain potency in vivo. EMBO J. 18:6439-6447.

20. Ye, Q. and H.J. Worman. 1995. Protein-protein interactions between human nuclear lamins expressed in yeast. Exp. Cell Res. 219:292-298.

21. Valenta, T., G. Hausmann, and K. Basler. 2012. The many faces and functions of $\beta$-catenin. EMBO J. 31:2714-2736.

22. Ma, L.Y., G. King, and L. Rothfield. 2003. Mapping the MinE site involved in interaction with the MinD division site selection protein of Escherichia coli. J. Bacteriol. 185:4948-4955.

23. Ren, B., K.J. Chee, T.H. Kim, and T. Maniatis. 1999. PRDI-BF1/Blimp-1 repression is mediated by corepressors of the Groucho family of proteins. Genes Dev. 13:125-137.

24. Tolkunova, E.N., M. Fujioka, M. Kobayashi, D. Deka, and J.B. Jaynes. 1998. Two Distinct Types of Repression Domain in Engrailed: One Interacts with the Groucho Corepressor and Is Preferentially Active on Integrated Target Genes. Mol. Cell. Biol. 18:2804-2814.

25. Grbavec, D. and S. Stifani. 1996. Molecular interaction between TLE1 and the carboxyl-terminal domain of HES-1 containing the WRPW motif. Biochem. Biophys. Res. Commun. 223:701-705.

26. Heimbucher, T., C. Murko, B. Bajoghli, N. Aghaallaei, A. Huber, R. Stebegg, D. Eberhard, M. Fink, et al. 2007. Gbx2 and Otx2 interact with the WD40 domain of Groucho/Tle corepressors. Mol. Cell. Biol. 27:340-351.

27. López-Ríos, J., K. Tessmar, F. Loosli, J. Wittbrodt, and P. Bovolenta. 2003. Six3 and Six6 activity is modulated by members of the groucho family. Development 130:185-195.

28. Eberhard, D., G. Jiménez, B. Heavey, and M. Busslinger. 2000. Transcriptional repression by Pax5 (BSAP) through interaction with corepressors of the Groucho family. EMBO J. 19:2292-2303.

29. Ishibashi, M., K. Moriyoshi, Y. Sasai, K. Shiota, S. Nakanishi, and R. Kageyama. 1994. Persistent expression of helix-loop-helix factor HES-1 prevents mammalian neural differentiation in the central nervous system. EMBO J. 13:1799-1805.

30. Scott, D.E., A.R. Bayly, C. Abell, and J. Skidmore. 2016. Small molecules, big targets: drug discovery faces the protein-protein interaction challenge. Nat. Rev. Drug Discov. 15:533550.

31. Vassilev, L.T., B.T. Vu, B. Graves, D. Carvajal, F. Podlaski, Z. Filipovic, N. Kong, U. Kammlott, et al. 2004. In vivo activation of the p53 pathway by small-molecule antagonists of MDM2. Science. 303:844-848.

Received 23 December 2016; accepted 09 March 2017.

Address correspondence to Thomas Czerny, Department of Applied Life Sciences, University of Applied Sciences, FH Campus Wien, HelmutQualtinger-Gasse 2, A-1030 Vienna, Austria. E-mail: thomas.czerny@fh-campuswien.ac.at

To purchase reprints of this article, contact: biotechniques@fosterprinting.com 